\title{
Design of a Mobile-App for Non-Invasively Detecting High Blood Cholesterol Using Eye Images
}

\author{
Yaser Alhasawi \\ Claremont Graduate University \\ yaser.alhasawi@cgu.edu
}

\author{
Balakrishnan Mullachery \\ Claremont Graduate University \\ alakrishnan.mullachery@cgu.edu
}

\author{
Prof. Samir Chatterjee \\ Claremont Graduate University \\ samir.Chatterjee@cgu.edu
}

\begin{abstract}
Medical research proves that high blood cholesterol is identifiable through the iris portion of the human eyes. Currently, a clinical blood test is the only method used in identifying high cholesterol. This study proposed an IT-based approach to prove the concept of monitoring cholesterol content in the blood using the human irises. We have developed a conceptual framework and a smartphone application aimed at capturing an image of the eye and analyzing it for symptoms associated with high blood cholesterol. This is a break-through innovation for a non-invasive way of finding out blood-cholesterol. The application captures the eye image and necessary metadata from a user. Then, a software code will examine the textural pattern of the eye, thus detecting the presence of high blood cholesterol. The result will be displayed on the user's mobile application. Primarily, the feedback to the user will be either "No" or "Yes" cholesterol content in the blood. The results presented through this app will form the basis for a further medical checkup if necessary.
\end{abstract}

Keywords: Health informatics, high blood cholesterol, mobile apps, image processing.

\section{Introduction}

A large population of people from all over the world suffers from a stroke, heart disease, and other cardiovascular ailments. According to the Center for Disease Control (CDC) and Prevention [1], one adult dies every 39 seconds as a result of these diseases. Moreover, the CDC has stated that for every six US dollars spent on healthcare, one dollar is spent treating patients suffering from these diseases. The primary cause of heart disease is high blood pressure and high cholesterol. However, it's hard to notice the signs of high cholesterol levels unless a qualified medical practitioner conducts a cholesterol test during a routine medical checkup. The inability to diagnose high cholesterol levels among individuals predisposes them to undue health risks. Medical researchers have proved that the human face shows observable symptoms of most diseases. For instance, the human eye, considered one of the most elaborate organs in the human body, is not only a window to the soul, but also manifests many types of illnesses in the body.

Studies have shown that changes in the human eyes signify potential health issues. Research on preliminary cholesterol detection has been carried out in various countries. According to Ramlee, R.A., Ranjit, S[2] and Sarika and Madhuri[3] regular eye examination is commonly used in detecting high cholesterol levels.

Just as thick plaques form in arteries, retinal vein occlusion is obvious in the eyes when blood flow is blocked. In other studies, an embolism is because of atherosclerotic plaques (cholesterol) which cause eye artery occlusion. Pfaffenbach and Hollenhorst argued that the presence of cholesterol in the blood results to several diseases including, but not limited to, both diabetes and high blood pressure [4].

The purpose of this study is to delve deeper into how technology can be used to examine the eye to 
detect high blood cholesterol. Although previous studies have indicated that high blood cholesterol can be detected by the human eye, there lacks an easily accessible and easy to use technology that can help health practitioners and other stakeholders to detect high cholesterol levels. In other words, the objective of this study is to present a solution to the market to allow people use the proposed technology to examine their eyes for cholesterol levels. Therefore, this study is important because it helps in implementing the theory of detecting high cholesterol levels through eye examination using a mobile application. The advent of technology, especially smartphones, has led to customization of most routine operations through a mobile application. Since most people have access to smartphones, this study highlights how people can use these gadgets to examine the cholesterol levels, hence minimizing risks associated with high cholesterol.

\subsection{Problem and research questions}

The inability to detect high blood cholesterol early can cause potential health risks. Earlier research showed that technology-based high cholesterol detection is widely based on the human eye $[5,6]$. Nevertheless, there lacks a readily available technology-based preliminary diagnosis for high cholesterol based on people's fingertips. Hence, we conducted this study by utilizing Smartphone and a cloud server to process eye images and identify potential eye textural pattern changes due to cholesterol in the human body and inform the user using the Smartphone in seconds. We have framed the study to answer three questions concerning cholesterol detection using information technology platform. They include:

What are the signs, symptoms and visible traits in the eyes that help to detect high blood cholesterol?

Is there a conceptual framework to develop a smartphone application for screening and processing eye image?

How can we validate and evaluate the correctnes s and effectiveness of this application?

\section{Literature review}

Various studies have shown the importance of eye examination as a basis for health determination $[4,5,6]$. Psychologists use eyes to tell the kind of emotions patients experience at any time [7]. Consequently, they can make a more credible judgment on patients' health and justification for their actions $[8,9,10]$. In other studies, eyes have been found to present paramount information on the levels of cholesterol in patients' blood.

According to Knabel and Chous (2015), the eye examination potentially offers better disease prognosis and management[11]. In fact, cardiovascular diseases and stroke could be best detected and promptly treated after proper eye exams. The authors further argued that presence of cholesterol in the blood is identified by an arcus around the outer layer of the cornea. Also, diseases such as diabetes, hypertension, thyroid and autoimmune diseases can be detected through eye examinations [11]. In previous studies, researchers used highly professional cameras to examine the eyes to determine whether someone had high cholesterol levels. On the contrary, this study advocates the use of smartphone camera to test the presence of high cholesterol. Therefore, in this paper, we relied on the smartphone camera to capture the eye image to detect the presence of high cholesterol. The availability of a smartphone application that could help eye examination to detect high cholesterol is a game changer. This will be the first mobile application to pre-detect cholesterol through the eye. Also, through this application, a new algorithm will be implemented to analyze the image from the eye deeply.

\section{Theoretical foundation}

The theoretical basis of this study draws upon "the Chronic Disease Indicator Framework"[12]. The Framework contains 41 indicators organized in a model comprised of 6 core domains: social and environmental determinants, early life/childhood risk and protective factors, behavioral risk and protective factors, risk conditions, disease prevention practices, and health outcomes/status.

The chronic illness indicator framework is important in grouping information in a chronological order necess ary for analys is and evaluation. On social and environmental determinants, education income and employment are provided. The second domain, which is early life/childhood risk and protective factor, breastfeeding, birth weight, exposure to smoke and family violence are provided. These provisions in the framework necessitate data entry. Therefore, the application platform, with the help of clouded data, fills in the framework with important information about the possible chronic diseases. The availability of cholesterol pigments in the eye confirms availability of the same in the blood. Furthermore, all six domains and their indicators need to be recorded 
for data analysis purposes. The environment around the patient is usually the most important indicator. It provides information on the most predisposed area for chronic diseases.

Therefore, this study conforms to the public health agency of various countries such as Canada in identifying possible indicators of chronic diseases. The information is used in predicting trends and possible predisposing factors for chronic diseases. Besides, the framework is important in developing public health interventions and evidenced information for decision-making processes. The framework presented through this research proved very efficient in documentation and analysis processes.

\section{Design and development of artifacts}

This study proposed a smartphone based eye image classification and analysis approach (CChecker) to identify the pattern of digital values of a pixel that corresponds to cholesterol presence in a human body. Image classification and pattern recognition were an important method that was developed for satellite remote sensing and medical imaging and improved in different contexts by Nivas and Manigandan (2015)[13]. We anticipated that the evidence of practical use-cases and workflows in solving real world problems should be included as an integral part of the solution. This was an important prediction regarding the practicality of the method in solving real world problems. The research methodology used in the study is a design science research principle. The different artifacts of the entire workflow and the substeps that constitute a single artifact and their relationships are identified in the workflow. Hevner et al. stated that the discovery of truth might lag the application of its utility and design science research paradigm which seeks to create "what is effective." So the efficacy of the workflow and its independent constituents are evaluated for its performance and scalability measures [14]. Another critical as sessment performed during the workflow development is the construction

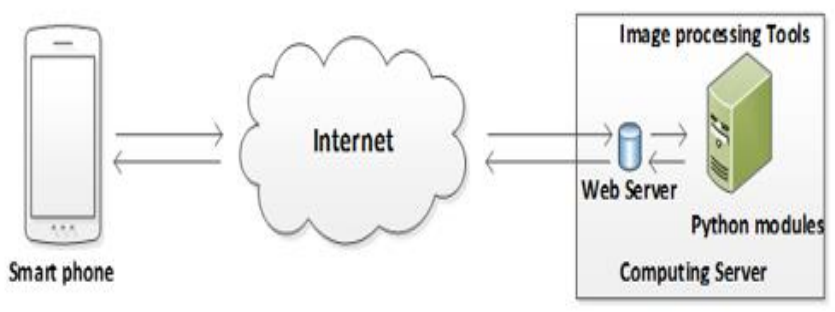

Figure 1. The proposed solution architecture

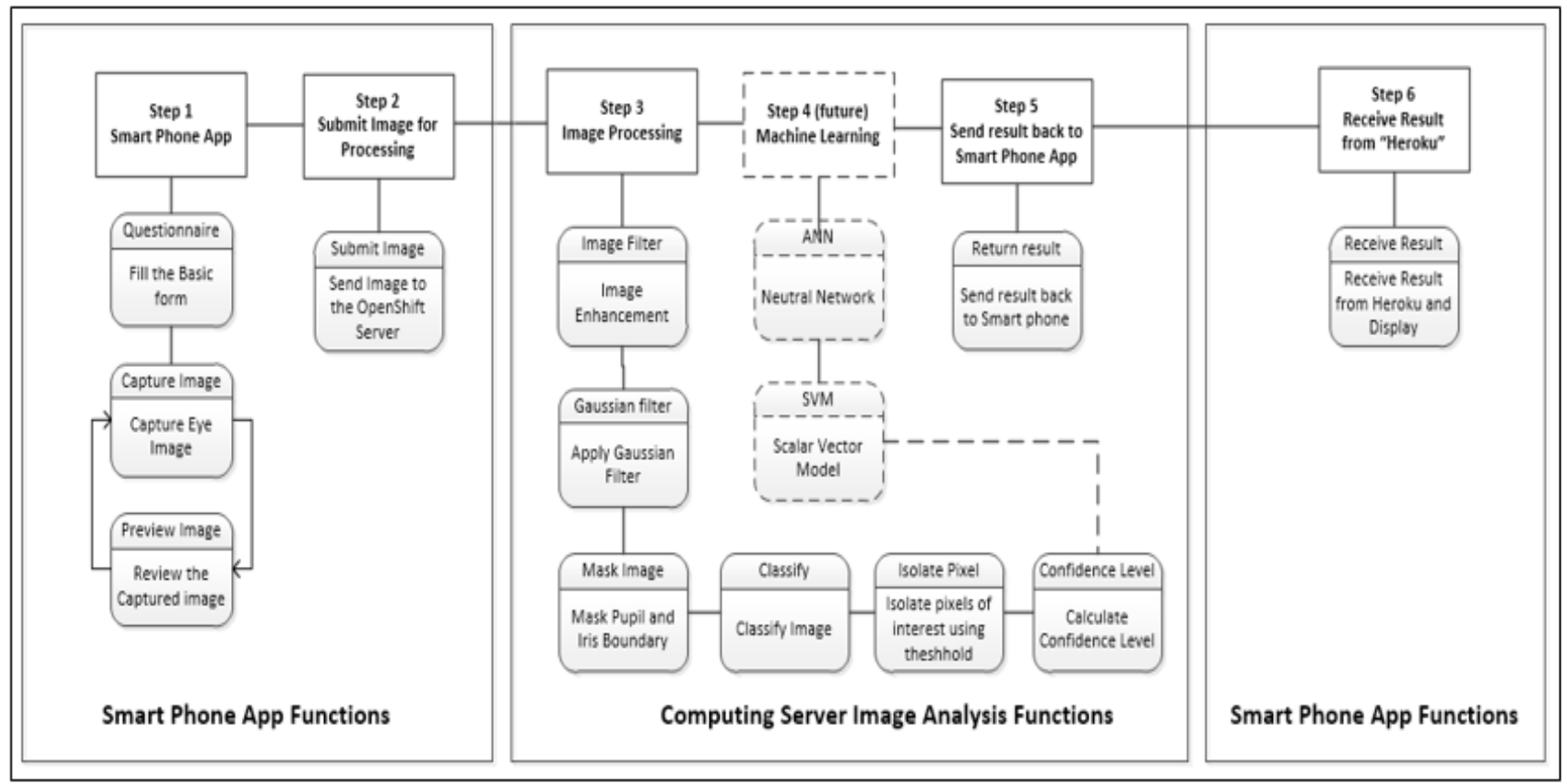

Figure 2. The software components and functions 
of a generic and complete working model. If any discovery or knowledge is identified, it is considered an additional process and plugged into an individual artifact without disturbing the overall workflow. This design approach will help the framework to be very agile and can be enhanced or expanded as needed. For instance, a predictive model using Machine Learning approach is included in the workflow architecture to improve the confidence level using multiple methods. However, the current study will not include Machine Learning. The proposed solution architecture, based on the identified problem, can be categorized as illustrated in Figure 1. such that: the Smartphone App is for data capture and reporting the result, while Cloud Computing Engine and Internet is for commutation between Smartphone and Image Processing Cloud Computing Engine. Based on these categories at a conceptual level, the solution will comprise the software components and functions illustrated in Figure 2.

\subsection{Artifact 1}

A smartphone application performs functions shown in Step 1, Step 2 and Step 6. This application will have four main functionalities. The metadata data will validate image analysis result within the algorithm as well as implement Machine Learning algorithms.

\subsubsection{Algorithm Steps}

First, Metadata gathering form. User input basic metadata such as "Age," "Height," "Weight," "Gender," "Cholesterol Medication Status," "Eating Habit," "Eye Color," and "Ethnic Background." Second, Capture eye (iris portion) image within a provided frame of reference. An iris image capturing tool: The tool will display a photo captur

ing a frame of reference (square). This frame will be the hard-coded size (height and width) of the image. There will be a circular ring that touches the four corners of the frame of reference with cross lines passing through the center of the circle. The radius of the circle is fixed and will be half of the image width or height. The user should be able to zoom to fit the iris, touching the circle exactly. During the photo capture, a user can use the cross-wire intersection to navigate and place the center of the pupil (or center of the iris).

Figure 3. below illustrates the configuration of the image capturing tool. A well-lighted environment or natural light should be used for image capture to avoid maximum light reflection from the eye. A flashlight will help to reduce other light sources that increase the reflection of the image. Then, an image preview function will be used to inspect the image before sending for processing visually. The user will have the option to re-capture the image using the tool. Fourth, upload the image to the computing server.

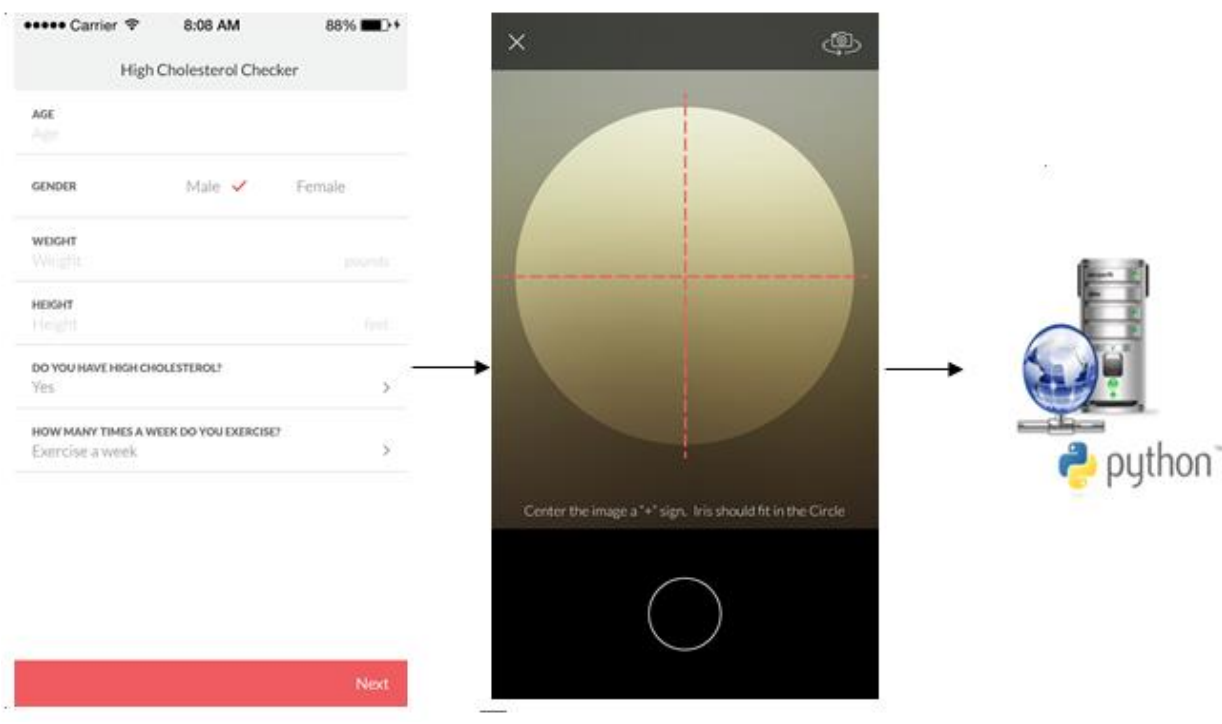

Figure 3. The image capturing tool 


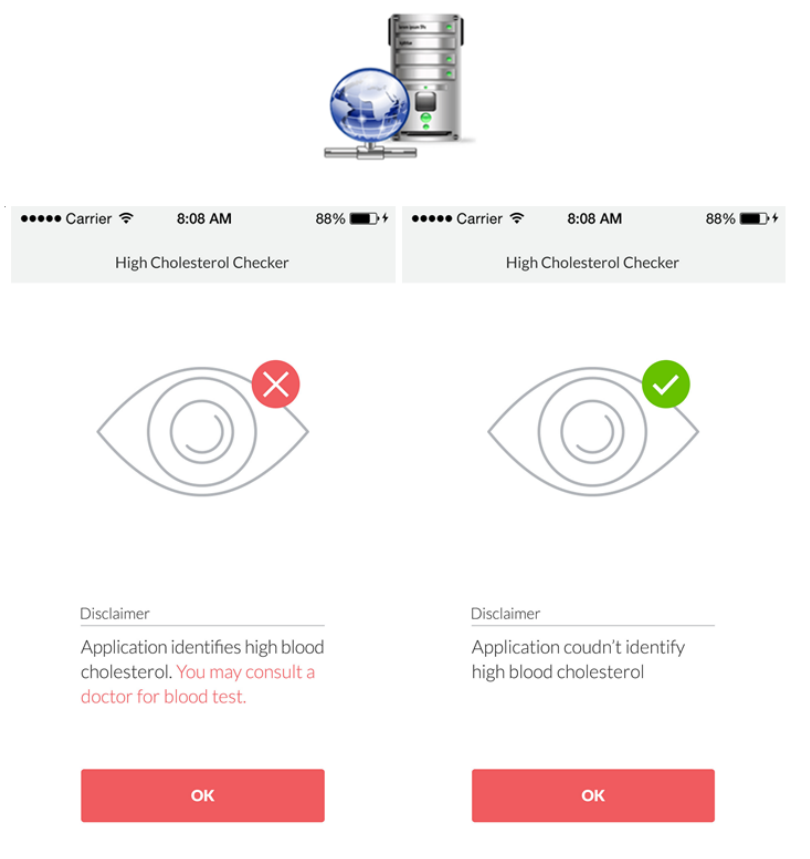

Figure 4. The result of the image analysis

(Step 2): The image will be uploaded to the Heroku server and run through the image processing tool. The last step (Step 6), includes receiving the result. The result of the image analysis process will be displayed on the Smartphone as either "Yes" or "No." If the application identifies high blood cholesterol, then "Yes" will be displayed in the red color circular background. Otherwise "No" will be displayed in the green circular background as illustrated in Figure 4. below. A disclaimer will be displayed in red text. For "Yes" - the disclaimer will be "Application identifies high blood cholesterol. You may consult a doctor for a blood test. On the other hand, a disclaimer for "No," will attest that the "Application couldn't identify high blood cholesterol."

\subsection{Artifact 2}

Computing server configured with Heroku internet server (IS) and Python-based image processing computing server. Heroku is a cloud platform for Smartphones used for connecting and running image processing algorithms

\subsubsection{Algorithm Steps}

- First, Install and configure Web server.

- Second, Install and configure Python modules Script, Scikit Learn, OpenCV, and Numpy.
- Third, Install and configure Cholesterol detecting custom tool.

\subsection{Artifact 3}

An Image Processing and Machine Learning software include a suite of automated processes; workflows, Machine Learning algorithms (future), rule- based data, validation process and tool for improving the data processing quality and consistency. It will cover Steps 3, 4 and 5.

\subsubsection{Algorithm Steps}

First, Image noise reduction and enhancement: A digital image is a collection of pixels in a matrix mode with rows and columns which are characterized by the light reflectance of an object. The intensity of the light reflectance is based on the textural properties and material content of the object. This information is stored in the image as a pixel value. In our algorithm, we are attempting to distinguish the pixels that represent the formation of cholesterol traces in the eye. However, there will be too many other noises that are likely to blur or make it hard to distinguish image characteristics using pixels. Most of the eye is supposed to have dark colored pixels. Our first attempt is to minimize the noises and smoothen the image. Then, replace cells in the raster 
based on the majority of their adjacent neighboring cells values to smoothen the image (Majority Filter). Next, rescale the pixel values using the Gaussian function, which transforms the input values through a normal distribution. When applying an increasing evaluation scale, the mid-point of the normal distribution defines the most preferred value. Lastly, the iris will be isolated using Hough Circle. Figure 5 (the eye image is borrowed from a third party location where confirmed high cholesterol content in the blood). illustrates the outcome of the tool.

Second, mask image outside the iris and inside the pupil: The study is focused on the area between pupil and the outer edge of the iris. The pupil will be masked because it contributes to the most imagerelated reflection. Then, the pixels are classified into two categories; namely, bright and dark pixel values. If the ratio between bright pixels to the total number of pixels is more than or equal to 5\%, the binary classification result is "Yes," which is otherwise "No." Fourth, Machine Learning algorithm (Python tool) (step 4). As already established, this tool is not included in the current study. Therefore, send the result back to the Smartphone App (Step 5): Communication back to the Smartphone with eye image analysis result "Yes" or "No."

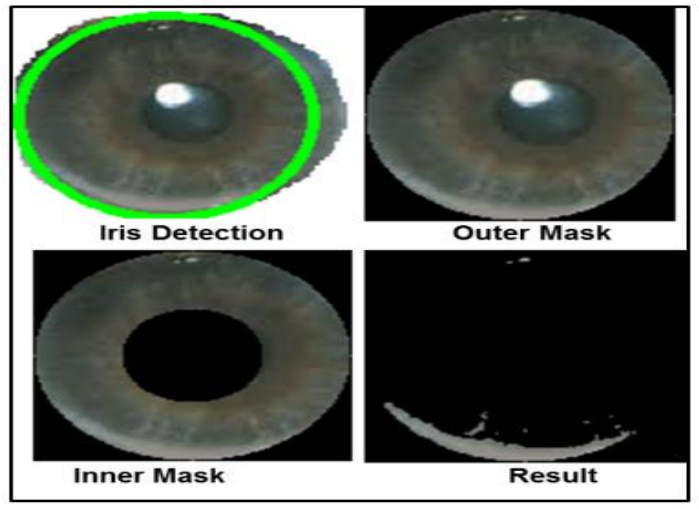

Figure 5. The outcome of the tool

\section{Evaluation of the Artifact}

The output of the application is a binary data classified as either "Yes" or "No." The entire workflow model is evaluated in a prototype project by running the application using datasets collected in two scenarios; namely,

Scenario 1: Capturing eye images of two people already identified with high blood cholesterol

Scenario 2: Capturing eye images of three individuals not identified with high blood cholesterol, yet.After processing the sample images, the results are inserted into a confusion matrix as illustrated in the Table - 1. A confusion matrix is a table used in describing the performance of a classification model on a set of test data with known true values. In our study, the result can be two values, either "Yes" or "No." "Yes" indicates that the eye image analysis process resulted with a high blood cholesterol in the study sample. On the other hand, "No" signifies that the eye image analysis process resulted with no high blood cholesterol in the study sample.

In Scenario 1, the tool correctly identified for cholesterol status after processing of all three images. The actual status cholesterol was "No" for all three images, and the solution predicted for all images are "No" cholesterol (TN = 3 and FP =0). The Actual Cholesterol No $=3$.

\section{Table 1. Confusion matrix}

\begin{tabular}{|c|c|c|c|}
\hline Total Sample ' $n$ ' $=\mathbf{5}$ & Predicted "No" & Predicted "Yes" & Total \\
\hline Actual "No" & TN=3 & FP=0 & 'Actual No' =3 \\
\hline Actual "Yes" & FN=1 & TP=1 & 'Actual Yes' = 2 \\
\hline Total & 'Predicted No' $=4$ & 'Predicated Yes' $=1$ & \\
\hline
\end{tabular}

In Scenario 2, after processing the two images, the tool correctly identified one image with cholesterol and another one without cholesterol. The actual status of cholesterol was "Yes" for both images, and the solution predicted for one image was "Yes, " and another one was "No" cholesterol $(\mathrm{FN}=1$ and $\mathrm{TP}=1)$. The Actual Cholesterol Yes $=2$. This information is inserted into a confusion matrix as illustrated in the Table - 1 and out of 5 images, four images were interpreted as cholesterol content "No" and one image interpreted as cholesterol "Yes" instead of three and two. Based on this numbers, accuracy, precision, specificity, and statistical sensitivity values are calculated. The confusion matrix and further statistics generated from the confusion matrix are validated and evaluated the correctness and effectiveness of this application.True Positives (TP): The tool predicted "Yes" (have the high blood cholesterol), and do have high blood cholesterol.

- True Negatives (TN): The tool predicted "No," but don't have high blood cholesterol. Figure 6. illustrates the outcome. 
- False Positives (FP): The tool predicted "Yes," but don't have high blood cholesterol. ("Type I error.") Figure 8. illustrates the outcome

- False Negatives (FN): The tool predicted "No," but do have the high blood cholesterol. ("Type II error.") Figure 7. illustrates the outcome.
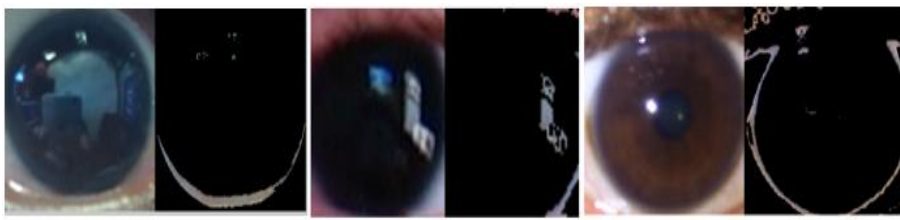

Figure 6. the tool predicted "No."

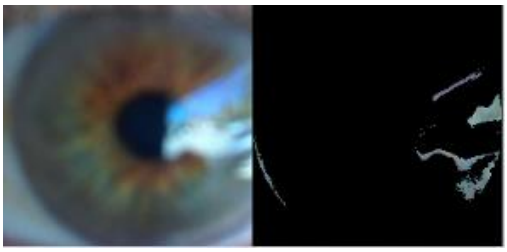

Figure 7. The tool predicted “No” ("Type II error.")

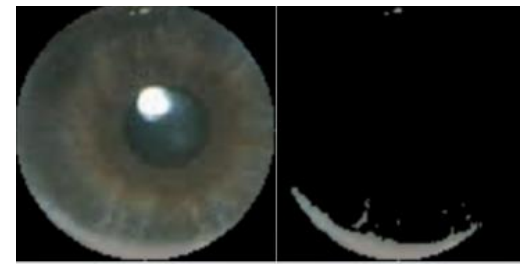

Figure 8. The tool predicted "Yes" ("Type I error")
The reason for one Type II error: The eye image Figure7. was collected from a person under cholesterol medication. Therefore, the prominence of the signal (the pixel value) thathe algorithm expects would be a weak signal. Also, this image was influenced by environmental factors such as light since it was not captured in a controlled environment. More detailed study is essential to enhance the algorithm to handle Type II error by enhancing the significance and improving the confidence level. In our next study, we will provide a more balanced approach to reducing the influence of external environmental factors and improve the confidence level by incorporating machine learning, hence reducing Type II error(s).

The total number of sample ("n"): Number of sample eye images processed using the tool. The above Metrics (Table 2.) are computed from a confusion matrix for a binary classifier. The accuracy is estimated to be 80 percentage and precision 100 percentage. The next to metrics table are True Positive Rate (y-axis) against the False Positive Rate (x-axis) which are graphically plotted using ROC Curve. The algorithms/tools that are part of every artifact will be refined by altering the various image classification and filter parameters such as classification pixel value ranges, majority filter resampling window size, etc. based on the evaluation findings. The prototype test is repeated three times to refine the workflow and to improve the output accuracy level. Based on the final metrics the three proposed research questions are discussed and concluded.

\begin{tabular}{|c|c|c|c|c|}
\hline Description & Count & Formula & Calculted Value & Percentage \\
\hline Total =N & 5 & & & \\
\hline True Negative = TN & 3 & & & \\
\hline False Positive = FP & 0 & & & \\
\hline Flase Negative = FN & 1 & & & \\
\hline True Positive = TP & 1 & & & \\
\hline Actutal No $=\mathrm{AN}$ & 3 & & & \\
\hline Actutal Yes = AY & 2 & & & \\
\hline Predicated No = PN & 4 & & & \\
\hline Predicated Yes = PY & 1 & & & \\
\hline Accuracy & & $(\mathrm{TP}+\mathrm{TN}) \mathrm{N}$ & 0.80 & $80.00 \%$ \\
\hline Error Rate & & $(\mathrm{FP}+\mathrm{FN}) / \mathrm{N}$ & 0.20 & $20.00 \%$ \\
\hline True Positive Rate & & TP/AY & 0.50 & $50.00 \%$ \\
\hline False Positive Rate & & FP/AN & 0.00 & $0.00 \%$ \\
\hline Specificity & & TN/AN & 1.00 & $100.00 \%$ \\
\hline Precision & & TP/PY & 1.00 & $100.00 \%$ \\
\hline Prevalence & & $\mathrm{AY} / \mathrm{N}$ & 0.40 & $40.00 \%$ \\
\hline
\end{tabular}

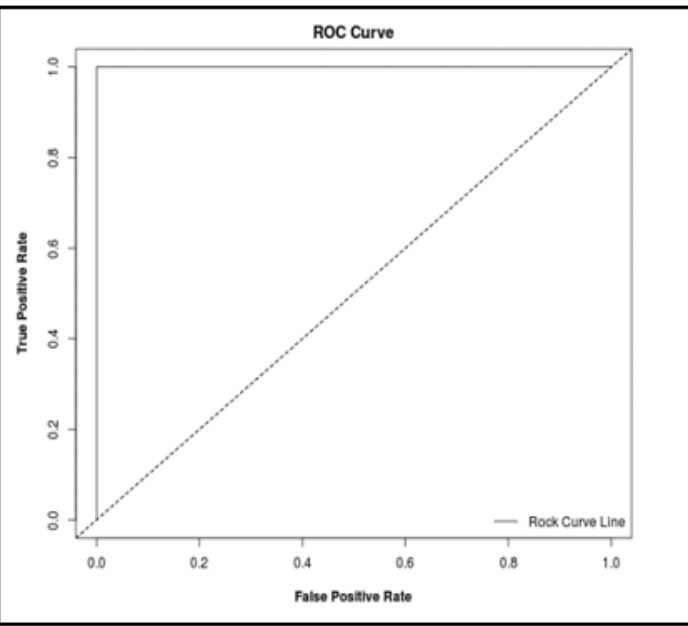

Table 2. Metrics 


\section{Discussions and Findings}

Despite the challenges of deploying the solution in a real-world application, our study provided an insight to the feasibility of a smartphone-based medical application. It alerts future health implication and makes potential patients aware of potential health risks and counsels them to consult medical checkup. In our initial evaluation of the solution using five eye images, we had 80 percent accuracy and 100 percent precision. One sample image reported as false negative (which could be valid, but needs more investigation in comparison with others) and reduced the accuracy. The negatively reported 'No" could be attributed to many factors. For instance, it is possible that the user that had been previously identified to have high cholesterol, presented with low cholesterol levels due to medication exercise and food restrictions. Therefore, there was a need to turn the threshold used in the tool. The negatively reported "No" could as well be caused by distortion of the eye image by various factors including camera, light, resolution, etc. However, $80 \%$ accuracy is considered an adequate result in an image analysis science. Typical binary classification ROC curve measurement is used for evaluation. The plotted ROC curve use specificity and sensitivity to provide maximum Area under Curve (AUC) value, which is also an indication of the positive evaluation result of the solution irrespective of the lack of sufficient input sample images. Evaluating the solution using more variety of images will provide efficacy of the solution, which will be our immediate future goal of this study.

The current study can be expanded into different disease-screening methods in the medical industry. Utilizing such devices for medical screening can make a huge difference in the healthcare system in terms of giving the right treatment at the right time for the patients. In most of the cases, patients are not aware of their health issues unless it became worse.

There are few challenges and implementation limitations we have experienced during the study. The quality of eye images is influenced by various technical and environmental factors such as camera performance and uncontrolled lights among others. The resolution and quality a camera on every smartphone available in the industry differ in characteristics. This will limit the development of a generic algorithm capable of accommodating all the technical specifications of the devices. Another contributing factor for image degradation is the environment in which the image is taken. We cannot control the users or the users may not be good in optics of the photography. The uncontrolled lights can diminish the image signals, which form important inputs in the algorithm. These factors will be studied and necessary enhancement in the proces s and algorithms developed in the ongoing research. Another major challenge identified was image classifications. based on the eye colors. The lessons learned in this study will help to include the necessary enhancements in the image analysis techniques, by leveraging machine learning and artificial neural network techniques in the ongoing study. One of the major practical implementation of this analysis is to minimize the false negative. Reliability of the results in terms of false negative versus false positive can be a practical limitation of this method. The percentage of false negative value can reduce trust in the practical application of this method in the medical field. In our ongoing studies, various methodologies including machine learning will be included to improve the reliability of the method.

\section{Conclusion}

Image analysis and interpretation are very popular in many scientific and medical applications. There are many image processing algorithms, and tools are available in a various format. The use of these techniques is purely a subjective approach. In those cases, the users manually perform appropriate image analysis software to get the information that he/she is interested in. However, in recent years, more applications have been developed to report the image analysis results using dashboard application. In our study, the fundamental requirement is to run the algorithm in an automated mode, without inspecting the input image. Therefore, the challenge is how to automatically run the image analysis algorithms in a generic approach with the right parameters used within the algorithm for different images. The scientific evaluation of this study requires many input eye image data with a different eye and image characteristics. Images can vary from people to people, camera conditions and lighting situation where the picture is taken. For the initial evaluation, we have used two groups of images. One group includes pictures of participants with cholesterol, and the other group is pictures of people without cholesterol. The first group we have two eye images and second group 3 images. This five image evaluation study helped us to include additional design changes in the proposed solution. For instance, we looked into how we could eliminate or minimize the influence of light reflections from the 
eye image to achieve ideal pixel classification threshold, which consequently controlled false negative and false positive. Also, we considered how images are classified according to different eye colors, how to improve the algorithm without missing a small portion of the iris in the image analysis, and how to minimize image distortion due to mobile camera handling. The answer to these questions will help in improving the usability of the proposed solution and its relevance in tackling real world problems. Most of these challenges can be accomplished by enhancing image analysis algorithm and implementing Machine Learning to improve image classification and leverage metadata for internal validation of data within the algorithm and Machine Learning, besides predictive analytic techniques. Finally, our research questions were answered through extensively searching readily available literature in the medical and IT research article databases. In our internet based research, we were unable to find any smartphone-based application to detect cholesterol from the eye. As part of the study, we developed a framework, developed and evaluated the feasibility of such application in helping people to monitor their cholesterol levels through a commonly available device and recommend appropriate cause of action, which answers the second question. For the last question, we have demonstrated a workflow and evaluated scenarios using confusion matrix and ROC curve which are excellent in evaluating the efficiency of a binary clas sification.

\section{References}

[1] High Blood Pressure and Cholesterol. - Google Search, https://www.google.com/\#q=High+Blood+Pressure+and+C holesterol.

[2] R.A. Ramlee, and S.Ranjit, "Using iris recognition algorithm, detecting cholesterol presence", In: Information Management and Engineering, International Conference, ICIME, 2009. pp. 714-717. IEEE (2009).

[3] G. Sarika, and S. Madhuri, "Automated detection of cholesterol presence using iris recognition algorithm" Int. J. Comput. Appl. 133, 41-45 (2016).

[4] D.D. Pfaffenbach, and R.W. Hollenhorst, "Morbidity and Survivorship of Patients by Embolic Cholesterol Crystals in the Ocular Fundus", Am. J. Ophthalmol. 75, 66-72 (1973).

[5] S. G. Songire, and Joshi, M. S. (2016). Automated Detection of Cholesterol Presence using Iris Recognition Algorithm. International Journal of Computer Applications (0975 - 8887) Volume 133 - No.6.

[6] Brzozka et al., 2017. Sensors and Actuators B: Chemical. An international journal devoted to research and development of chemical transducers. Volume 246, Pages 1-1116.
Knabel, T.L.: Impact of Eye Exams in Identifying Chronic Conditions.

[7] B. L. Fredrickson, "The Role of Positive Emotions in Positive Psychology: The Broaden-and-Build Theory of Positive Emotions", Am Psychol, 56(3), 2001, p. 218-226.

[8] I. B. Mauss, and M. D. Robinson, "Measures of emotion: A review", Cogn Emot, 23(2), 2009, p. 209-237. doi: 10.1080/02699930802204677

[9] E. Jeffe, "The Psychological study of smiling", Association for Psychological Science, (2010) https://www.psy chologicalscience.org/observer/thepsychological-study-of-smiling\#.WTeJvuuGPIU

[10] D. Matsumoto, and H. S. Hwang, "Psychological Science Agenda: Reading facial expressions of emotion", American Psychological Association (2011).

[11] T. L. Knabel, and L. M. Chous, "Impact of Eye Exams in Identifying Chronic Conditions", UnitedHealthCare, 2015

https://www.uhc.com/content/dam/uhcdotcom/en/Employer s/PDF/EyeExamsChronicConditions.pdf

[12] M.T. Betancourt, K.C. Roberts, T.L. Bennett, E.R., Driscoll, G. Jay araman, and L. Pelletier, "Monitoring chronic diseases in Canada: the Chronic Disease Indicator Framework. Mal. Chron", Blessures Au Can. 34, (2014).

[13] D.R. Nivas, and, T. Manigandan, "Classification of Human Eye Image using different Methods for Medical Applications", International Journal of Advanced Research in Computer and Communication Engineering. Vol. 4, Issue 4 (2015).

http://www.ijarcce.com/upload/2015/april-

15/IJARCCE\%2035.pdf.

[14] R.H. Von Alan, S.T. March, J. Park, and S. Ram, "Design science in information systems research", MIS Q. 28, 75-105 (2004).

[15] D.C. Duro, S.E. Franklin, and M.G. Dubé, "A comparison of pixel-based and object-based image analysis with selected machine learning algorithms for the classification of agricultural landscapes using SPOT-5 HRG imagery", Remote Sens. Environ. 118, 259-272 (2012). 